\title{
Analysis of anti-RNA polymerase III antibodies in Chinese Han systemic sclerosis patients
}

\author{
Chenxi Liu ${ }^{1} \cdot$ Yong Hou ${ }^{2}$ • Dong Xu ${ }^{2} \cdot$ Liubing $\mathrm{Li}^{1} \cdot$ Yanfang Zhang ${ }^{1} \cdot$ Linlin Cheng ${ }^{1} \cdot$ Songxin Yan ${ }^{1}$. \\ Fengchun Zhang ${ }^{2} \cdot{\text { Yongzhe } \mathrm{Li}^{1}}^{1}$
}

Received: 26 May 2019 / Revised: 12 September 2019 / Accepted: 2 October 2019 / Published online: 19 December 2019

(C) The Author(s) 2019

\begin{abstract}
Objectives This study aimed to assess the prevalence and clinical correlation of anti-RNA polymerase III antibodies (anti-RNAP III) in Chinese Han systemic sclerosis (SSc) patients.

Methods Serum samples from 236 patients with SSc, 125 patients with connective tissue diseases (CTD), and 166 healthy controls (HCs), recruited from Peking Union Medical College Hospital and 21 other medical centers in China, were tested for antibodies to RNA polymerase III by means of a line immunoassay (LIA) or an enzymelinked immunosorbent assay (ELISA) kit.

Results Anti-RNAP III antibodies were found in 14/236 SSc patients (5.93\%), 1/125 (0.80\%) CTD patients, and $0 / 166(0.00 \%)$ HCs. The prevalence of anti-RNAP III was higher in SSc patients than in the CTD and HC groups ( $p$ $=0.02, p=0.001$, respectively). Renal crisis was significantly more common in patients with anti-RNAP III than patients without anti-RNAP III (42.9 vs. 4.1\%, $p<0.0001)$. Gastrointestinal involvement was significantly more common in patients without anti-RNAP III than patients with anti-RNAP III (53.6 vs. 21.4\%, $p=0.039$ ). There was good agreement between the ELISA and line immunoassay (LIA) detection capabilities for anti-RNAP III.

Conclusions The anti-RNAP III antibody, which was detected by ELISA, has diagnostic value for SSc and predictive value for SSc-related renal crisis. Both ELISA and LIA are very reliable methods for anti-RNAP III.

Key Points

- The prevalence of anti-RNAP III antibody was determined in Chinese SSc patients and performed ethnic differences.

- The clinical association between anti-RNAP III antibody and Chinese SSc patients was evaluated in this research.

- Methodological consistency of detection of anti-RNAP III antibody using commercial ELISA and LIA methods was evaluated in this research.
\end{abstract}

Keywords Autoantibodies · Connective tissue disease $\cdot$ Enzyme-linked immunosorbent assay $\cdot$ RNA polymerase III $\cdot$ Systemic sclerosis

This research has previously been published as an abstract in conference proceedings in the 13th National Congress of Immunology of China. Liu, C. and Li. Y. Anti - RNA polymerase III antibodies in Chinese Han systemic sclerosis patients detected by ELISA in Summary of the 13th National Congress of Immunology 2018.

Fengchun Zhang

zhangfccra@aliyun.com

Yongzhe Li

yongzhelipumch@126.com

1 Department of Clinical Laboratory, Peking Union Medical College Hospital, Peking Union Medical College, Chinese Academy of Medical Science, 1 Shuaifuyuan, Dongcheng District, 100730 Beijing, People's Republic of China
Department of Rheumatology and Clinical Immunology, Peking Union Medical College Hospital, Chinese Academy of Medical Sciences \& Peking Union Medical College, Key Laboratory of Rheumatology and Clinical Immunology, Ministry of Education, 1 Shuaifuyuan, Dongcheng District, 100730 Beijing, People's Republic of China 


\section{Introduction}

Systemic sclerosis ( $\mathrm{SSc}$ ) is an autoimmune disease characterized by vasculopathy, tissue fibrosis, and the production of autoantibodies $[1,2]$. The clinical manifestations of SSc are patient-specific, and most SSc patients will develop skin thickening with variable organ involvement, including interstitial lung disease (ILD), pulmonary arterial hypertension $(\mathrm{PAH})$, and renal crisis [3-5]. As abnormal immunity is activated, multiple autoantibodies can be detected in the serum of SSc patients, such as anti-nuclear antibodies (ANA), antitopoisomerase I antibodies (ATA, also known as anti-Scl-70 antibodies), and anti-centromere antibodies (ACA). Antitopoisomerase I antibodies are classically associated with ILD and the diffuse cutaneous form of SSc, while anticentromere antibodies are typically associated with $\mathrm{PAH}$ and the limited cutaneous form of SSc [6].

Anti-RNA polymerase III antibody (anti-RNAP III) is the third type of antinuclear autoantibody detected in patients with SSc. It was first shown to be associated with SSc in 1993 when Okano et al. discovered the anti-RNAP III antibody using radioimmunoprecipitation technology [7]. The RNA polymerase III antibody is a multi-protein complex that is located in the nucleoplasm. RP155 was identified as the major epitope of RNA polymerase III recognized by SSc serum [8]. In recent years, foreign studies have identified that the prevalence of anti-RNAP III in SSc ranges from 10 to $25 \%$ in American SSc patients [9, 10], nearly $6 \%$ in Japanese SSc patients [11, 12], 15.3\% in Australian SSc patients [13], and $19.4 \%$ in Canadian SSc patients [14], which has been linked to ethnic variations. Currently, there are few reports on the prevalence of anti-RNAP III in Chinese SSc patients and its correlation with the clinical features of SSc. Hence, we aimed to evaluate the prevalence and predictive value of anti-RNAP III in Chinese Han SSc patients.

\section{Materials and methods}

\section{Patients}

The study population included a total sample of 527 patients, including $236 \mathrm{SSc}$ patients (28 males and 208 females, mean age $48.3 \pm 12.5$ years) who fulfilled the 2013 ACR/EULAR classification criteria for SSc. ILD was diagnosed by the highresolution computed tomography in the SSc patients. PAH was diagnosed by mean pulmonary pressure values $\geq 25 \mathrm{mmHg}$ in SSc patients using the right heart catheterization. Gastrointestinal involvement related to SSc included reflux esophagitis, esophageal dysmotility, dysphagia, and others. Cardiac involvement included SSc-induced systolic or diastolic dysfunction. Renal crisis was defined as severe hypertension and rapid renal insufficiency. Some data about clinical manifestations were missing from the medical records. In addition, 30 patients were unclassified due to missing clinical data.

In addition, 125 control serum samples (mean age $46.0 \pm$ 15.5 years, matched for age and sex with SSc patients) were obtained from 25 primary Sjögren's syndrome (pSS) patients who fulfilled the American-European consensus group classification criteria [15], 25 rheumatoid arthritis (RA) patients who fulfilled the 1987 American College of Rheumatology (ACR) revised classification criteria [16], 25 systemic lupus erythematosus (SLE) patients who fulfilled the 1997 ACR revised classification criteria [17], and 25 dermatomyositis (DM) and 25 polymyositis (PM) patients diagnosed according to the Bohan and Peter criteria $[18,19]$, were studied. Also, 166 healthy subjects (mean age $47.6 \pm 12.5$ years, matched for age and sex with SSc patients) admitted to the Peking Union Medical College Hospital Health Examination Center for physical examination were also recruited for this study. All serum samples were stored at $-80^{\circ} \mathrm{C}$ until measurement. All the samples were obtained from patients recruited from the Peking Union Medical College Hospital (PUMCH) and other 21 health centers in China. The study was approved by the Ethics Committee of the Peking Union Medical College Hospital, and all recruited participants gave informed consent.

\section{Measurement of anti-RNAP III}

We used an anti-RNA polymerase III enzyme-linked immunosorbent assay (ELISA) kit for IgG (Medical and Biological Laboratories (MBL) Co. Ltd, Nagano, Japan) to retrospectively analyze 527 serum samples. The antigen was the recombinant immunodominant epitope based on the RP155 subunit of RNA polymerase III. The assay was performed according to the manufacturer's instructions. The values that were $\geq 28$ $\mathrm{U} / \mathrm{ml}$ were considered positive for anti-RNAP III. In addition, we also used a line immunoassay (LIA) to analyze anti-RNAP III with a commercially available kit (Euroline Systemic Sclerosis Nucleoli Profile IgG kit, Euroimmun AG, Lübeck, Germany) in $236 \mathrm{SSc}$ patient serum samples. The assay was performed according to the manufacturer's instructions. The results (negative, borderline, positive, and strong positive) were interpreted using the EUROScan software (Euroimmun AG, Lübeck, Germany), and borderline was considered as negative in this study.

\section{Statistical analysis}

The data were analyzed by SPSS version 19.0 for Windows (SPSS Inc, Chicago, IL, USA). Figure 1 was generated by GraphPad Prism 7.0 (La Jolla, CA). The frequencies of categorical variables were compared using Pearson's c2 or Fisher's exact test, when appropriate. Spearman correlation analysis was used to assess the relationship of anti-RNAP III levels using ELISA with anti-RNAP III levels using LIA. The 
Fig. 1 Prevalence of anti-RNAP III antibodies in SSc patients, CTD patients, and HCs. SSc systemic sclerosis, Anti-RNAP III anti-RNA polymerase III, CTD connective tissue diseases, $\mathrm{HCs}$ healthy controls

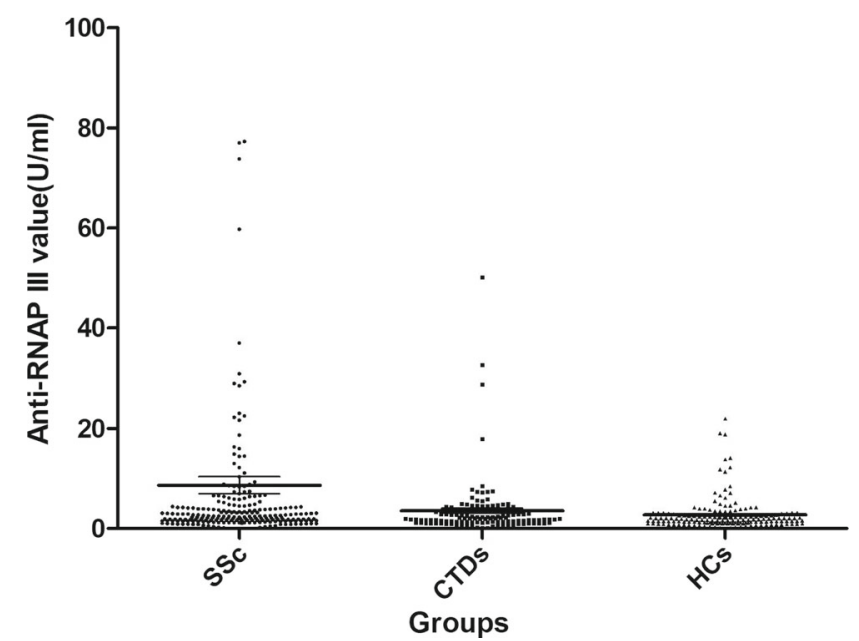

$\mathrm{SSc}=$ systemic sclerosis; Anti-RNAP III = Anti-RNA polymerase III; CTD = connective tissue diseases; HCs = healthy controls sensitivity, specificity, positive predictive values (PPV), negative predictive values (NPV), positive likelihood ratios (LR+ ), and negative likelihood ratios (LR-), were calculated accordingly. $p$ values that were less than 0.05 were considered statistically significant.

\section{Results}

\section{Prevalence of anti-RNAP III antibodies in various groups}

Serum samples from $236 \mathrm{SSc}$ patients, $125 \mathrm{CTD}$ controls, and 166 healthy controls were analyzed for anti-RNAP III by an MBL ELISA (Fig. 1). The prevalence of anti-RNAP III antibodies in the SSc, CTD, and HC groups were 5.93\% (14/236), $0.80 \%(1 / 125)$, and $0.00 \%(0 / 166)$, respectively. The prevalence of anti-RNAP III was significantly higher in SSc, compared with the CTD group $(p=0.020)$. When comparing the SSc with HC groups, the prevalence of anti-RNAP III was significantly higher in $\operatorname{SSc}(p=0.001)$, suggesting that antiRNAP III is useful for diagnosing SSc. The sensitivity and specificity of anti-RNAP III by the MBL ELISA in Chinese SSc patients were 5.93 and $99.2 \%$, respectively. The PPV and NPV were 93.4 and $52.5 \%$, respectively. The LR+, a multiple between the probability and a true positive judgement to the probability of a false positive judgment, was 7.41, and LRwas 0.95 . The high $L R+$ demonstrated that the accuracy of the ELISA results for anti-RNAP III is high.

\section{Clinical characteristics of SSc patients and clinical associations of anti-RNAP III}

The clinical characteristics of SSc patients and associations of anti-RNAP III are summarized in Table 1. Patients with anti-
RNAP III were not different from patients without anti-RNAP III considering gender (female, 78.6 vs. $88.7 \%$ ) and disease subtype (diffuse cutaneous, 50 vs. $37.1 \%$ ). No significant differences were discovered for disease duration (11.2 $\pm 8.1 \mathrm{vs.}$ $11.4 \pm 5.8, p=0.87$ ), joint involvement (35.7 vs. $50.5 \%, p=$ 0.427 ), ILD ( 50.0 vs. $67.1 \%, p=0.307$ ), PAH (35.7 vs. $19.8 \%, p=0.279$ ), or cardiac involvement (42.9 vs. $34.7 \%$, $p=0.739)$ in the patients with anti-RNAP III and without antiRNAP III, respectively. Patients with anti-RNAP III were older than patients without anti-RNAP III $(55.4 \pm 8.2$ vs. $47.9 \pm 12.9, p=0.005$ ). Renal crisis was significantly more common in patients with anti-RNAP III than patients without anti-RNAP III ( 42.9 vs. $4.1 \%, p<0.0001)$. The odds ratio (OR) was 17.8 with a $95 \%$ confidence interval of 5.1 to 62.0. Gastrointestinal involvement was significantly more common in patients without anti-RNAP III than patients with anti-RNAP III (53.6 vs. $21.4 \%, p=0.039$ ).

\section{Evaluation of anti-RNAP III in predicting clinical manifestations of SSC}

Anti-RNAP III was evaluated on its ability to predict the clinical manifestations of SSc (Table 2). Anti-RNAP III had a low PPV and NPV, especially when considering renal involvement (NPV 95.95\%). In addition, the LR+ and nLR- were universally low except for renal crisis (LR+ 11.05).

\section{Agreement between ELISA and LIA of detection with anti-RNAP III}

There was a good agreement between ELISA and LIA of detection with anti-RNAP III (Table 3). The $\kappa$ was 0.799 , representing a significant consistency. The $\chi 2$ was 144.26 , and the $p$ value was less than 0.001 , suggesting that the relevance of these two methods is very significant. The anti- 
Table 1 Clinical characteristics of SSc patients and clinical associations of anti-RNAP III

\begin{tabular}{|c|c|c|c|c|c|}
\hline \multirow[t]{2}{*}{ Characteristics } & \multicolumn{2}{|c|}{$\begin{array}{l}\text { Anti-RNAP III } \\
+(n=14)\end{array}$} & \multicolumn{2}{|c|}{$\begin{array}{l}\text { Anti-RNAP } \\
\text { III }-(n=222)\end{array}$} & \multirow[b]{2}{*}{$p$ value } \\
\hline & Total $^{\mathrm{a}}$ & $n(\%)$ & Total & $n(\%)$ & \\
\hline \multicolumn{6}{|l|}{ Gender } \\
\hline Female & 14 & 11 (78.6\%) & 222 & $\begin{array}{l}197 \\
(88.7 \%)\end{array}$ & 0.475 \\
\hline Male & 14 & $3(21.4 \%)$ & 222 & $25(11.3 \%)$ & \\
\hline Age (years) & 14 & $55.4 \pm 8.2$ & 222 & $47.9 \pm 12.9$ & 0.005 \\
\hline $\begin{array}{l}\text { Disease duration } \\
\quad \text { (years) }\end{array}$ & 14 & $11.2 \pm 8.1$ & 222 & $11.4 \pm 5.8$ & 0.87 \\
\hline \multicolumn{6}{|l|}{ Disease subtype } \\
\hline Diffuse & 12 & $6(50 \%)$ & 194 & $72(37.1 \%)$ & 0.558 \\
\hline Limited & 12 & $6(50 \%)$ & 194 & $122(62.9 \%)$ & \\
\hline \multicolumn{6}{|l|}{ Clinical manifestations } \\
\hline Joint involvement & 14 & $5(35.7 \%)$ & 222 & $112(50.5 \%)$ & 0.427 \\
\hline ILD & 14 & $7(50 \%)$ & 222 & $149(67.1 \%)$ & 0.307 \\
\hline PAH & 14 & $5(35.7 \%)$ & 222 & $44(19.8 \%)$ & 0.279 \\
\hline Gastrointestinal involvement & 14 & $3(21.4 \%)$ & 222 & $119(53.6 \%)$ & 0.039 \\
\hline Cardiac involvement & 14 & $6(42.9 \%)$ & 222 & $77(34.7 \%)$ & 0.739 \\
\hline Renal crisis & 14 & $6(42.9 \%)$ & 222 & $9(4.1 \%)$ & $<0.0001$ \\
\hline
\end{tabular}

SSc systemic sclerosis, Anti-RNAP III anti-RNA polymerase III, ILD interstitial lung disease, PAH pulmonary arterial hypertension

${ }^{a}$ Total number of SSc patients whose data were available for analysis

RNAP III values using ELISA in SSc patients had a significantly positive correlation with anti-RNAP III values using LIA (Fig. $2 ; r=0.824, p<0.0001$ ).

\section{Discussion}

This study aimed to determine the prevalence of antiRNAP III in Chinese SSc patients. The reported data of pooled prevalence of anti-RNAP III was $11 \%$ (95\% CI 8 to $14 \%$ ) in a transnational meta-analysis [20], which was consistent with the findings from Koenig et al.
[21]. However, our data was slightly lower with only $5.93 \%$ of Chinese SSc patients expressing anti-RNAP III because of the racial variation. From these data, we believe that the prevalence of anti-RNAP III is higher in Caucasian SSc patients and lower in Asian SSc patients, especially in patients from China and Japan.

Anti-RNAP III is known to be associated with some clinical manifestations in SSc, including diffuse cutaneous involvement and renal crisis [6]. Furthermore, Nikpour et al. [13] reported that anti-RNAP III was associated with diffuse disease, renal crisis, joint contractures, and higher modified Rodnan skin scores $(p<0.0001)$. Patients with anti-RNAP III

Table 2 Evaluation of anti-RNAP III in predicting clinical manifestations in SSc

\begin{tabular}{|c|c|c|c|c|c|c|}
\hline & $\operatorname{SEN}(\%)$ & $\operatorname{SPE}(\%)$ & PPV (\%) & NPV $(\%)$ & LR+ & LR- \\
\hline Diffuse subtype & 7.69 & 95.31 & 50.00 & 62.89 & 1.64 & 0.97 \\
\hline Limited subtype & 4.69 & 92.31 & 50.00 & 37.11 & 0.61 & 1.03 \\
\hline Joint involvement & 4.27 & 92.44 & 35.71 & 49.55 & 0.57 & 1.04 \\
\hline ILD & 4.49 & 91.25 & 50.00 & 32.88 & 0.51 & 1.05 \\
\hline PAH & 10.20 & 95.19 & 35.71 & 80.18 & 2.12 & 0.94 \\
\hline Gastrointestinal involvement & 2.46 & 90.35 & 21.43 & 46.40 & 0.25 & 1.08 \\
\hline Cardiac involvement & 7.23 & 94.77 & 42.86 & 65.32 & 1.38 & 0.98 \\
\hline Renal crisis & 40.00 & 96.38 & 42.86 & 95.95 & 11.05 & 0.62 \\
\hline
\end{tabular}

$S S c$ systemic sclerosis, $P A H$ pulmonary arterial hypertension, $S E N$ sensitivity, $S P E$ specificity, $P P V$ positive predictive value, $N P V$ negative predictive value, $L R+$ positive likelihood ratio, $L R-$ negative likelihood ratio 
Table 3 Agreement between ELISA and LIA of detection with anti-RNAP III

\begin{tabular}{lccccccrr}
\hline Detection method & TP, $n$ & TN, $n$ & FP, $n$ & FN, $n$ & Agreement, $n / n(\%)$ & $\chi 2$ & $\kappa$ & $p$ value \\
\hline ELISA vs. LIA & 13 & 217 & 6 & 0 & $230 / 236(97.5 \%)$ & 144.26 & 0.799 \\
\hline
\end{tabular}

$T P$ true positive, i.e., ELISA positive and LIA positive; $T N$ true negative, i.e., ELISA negative and LIA negative; $F P$ false positive, i.e., LIA positive and ELISA negative; $F N$ false negative, i.e., LIA negative and ELISA positive; ELISA enzyme-linked immunosorbent assay; LIA line immunoassay

had a higher risk of malignancies diagnosed within 5 years of the onset of the SSc skin disease. Motegi et al. [11] also reported the diagnostic usefulness and risk stratification of antiRNAP III for severe skin sclerosis and renal crisis. Steen [22] found that SSc patients with anti-RNAP III had a lower frequency of severe ILD. In this study, we found a significant difference in the frequency of renal crisis and gastrointestinal involvement between the patients with and without antiRNAP III. However, there was no association found with the diffuse cutaneous form as well as other clinical manifestations in our study. Probably the predictive power of antiRNAP III antibodies was limited by the low sensitivity, except for renal crisis, as expected.

Recent studies have shown that cardiopulmonary and kidney involvement accounts for most SSc-related deaths with ILD and PAH being the primary causes of deaths [23, 24]. Interestingly, anti-RNAP III was not associated with ILD or PAH. Therefore, the early diagnosis and treatment is essential for improving the prognosis of patients with SSc.

It has been reported that $2-15 \%$ of patients with SSc will develop renal crisis, and the SSc patients with renal crisis have 5-year survival rates of 50-60\% [25]. Patients with scleroderma renal crisis may develop malignant hypertension, anuria, and renal failure. These severe complications exacerbate the disease progression and lead to death in some cases. In our study, SSc patients with anti-RNAP III had a higher risk of renal crisis than patients without anti-RNAP III, with an OR of 17.8 and 95\% confidence interval 5.1 to 62.0 . In the SSc patients, the frequency of renal crisis was $15 / 236$ (6.4\%), which increased to $6 / 14$ $(42.9 \%)$ in the anti-RNAP III-positive SSc patients. The LR+ was 11.05 and the anti-RNAP III showed good predictive value for renal crisis. For SSc patients who are anti-RNAP III-positive, physicians should be alerted to the possibility of patients developing renal crisis. In return, the patients can receive timely treatment to avoid high-dose hormone therapy. Therefore, SSc patients positive for anti-RNAP III should regularly monitor their blood pressure, creatinine levels, and urine protein levels. For those SSc patients showing signs of renal crisis, treatment with an angiotensin-converting enzyme inhibitor (ACEI) can lead to improved prognoses [26]. However, despite the controversy of the finding, there has been reported that treatment with ACEI may be associated with an increased risk of dialysis or death before the abrupt rise in blood pressure and creatinine levels [27]. Notably, corticosteroid is commonly used for the treatment of the inflammatory symptom in SSc, but it is a risk factor for renal crisis[28]. Some studies have reported that SSc patients treated with moderate-to-high doses of corticosteroids performed an increased risk of renal crisis[29]. It is crucial to limit the corticosteroids use to low dose and the short duration possible.

As we all know, the immunoprecipitation (IP) assay is the gold standard for detecting anti-RNAP III antibodies. Previously, it was reported that the highly sensitive and specific ELISA system showed similar results to those of the IP assay [30]. While the agreement between the detection values from
Fig. 2 Correlation between ELISA and LIA of detection with anti-RNAP III among SSc patients $(n=236)$. $S S c$ systemic sclerosis, ELISA enzyme-linked immunosorbent assay, LIA line immunoassay

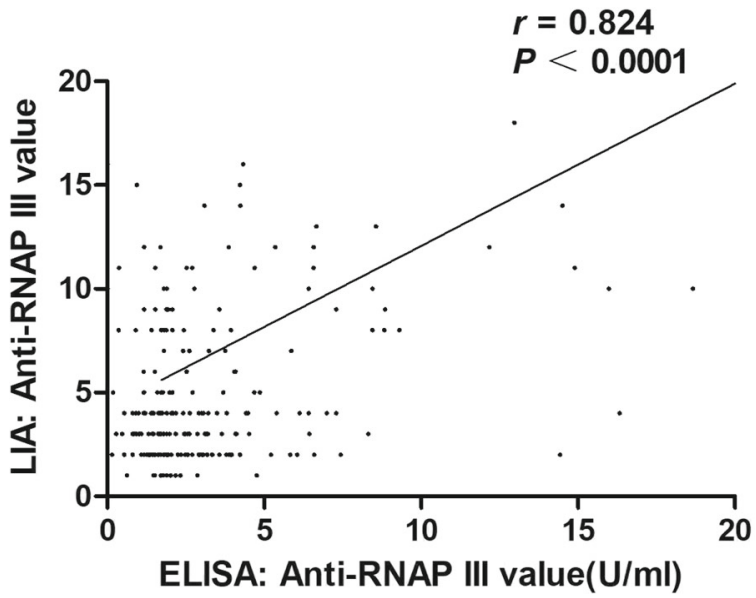

$\mathrm{SSc}=$ systemic sclerosis; ELISA = enzyme-linked immunosorbent assay; LIA = line immunoassay 
ELISA and LIA of detection was high $(\kappa=0.799)$, there were still several false-positive results with the LIA kit, suggesting that the assay needs to be improved before its clinical use.

In this study, gastrointestinal involvement was significantly more common in patients without anti-RNAP III than patients with anti-RNAP III. Previously, Ingraham et al. [31] reported an association between anti-RNAP III and gastric antral vascular ectasia (GAVE), another type of gastrointestinal involvement related to SSc, and the absence of anti-Scl-70 was useful for identifying the risk of developed SSc-induced GAVE. This finding was later verified by another research group [32]. However, Hung et al. [33] did not find the association between anti-RNAP III and GAVE, but they confirmed the negative association between anti-Scl-70 and GAVE. Thus, it can be seen that the results can vary between groups, suggesting that more rigorous multi-center large population trials are needed to investigate the relationship between anti-RNAP III and gastrointestinal diseases. In conclusion, the ELISA-detected antiRNAP III antibody has diagnostic value for SSc and predictive value for SSc-related renal crisis.

However, there were some limitations in the present study. Some data about clinical manifestations were insufficient, so the pulmonary function tests (PFTs) and Modified Rodnan Skin Score (MRSS) could not be provided because of the characteristics of retrospective study.

In conclusion, a commercial enzyme-linked immunosorbent assay (ELISA) kit for IgG was used to evaluate the diagnostic value of anti-RNA polymerase III in Chinese SSc patients in this study. There was a good agreement between ELISA and LIA of detection with anti-RNAP III. The ELISA-detected anti-RNAP III antibody has diagnostic value for SSc and predictive value for SSc-related renal crisis.

Acknowledgements We would like to thank all the patients with SSc recruited in this study, and multiple centers in China for the use of SSc sample and clinical data. This research was supported by grants from the Research Special Fund for Public Welfare Industry of Health (201202004), the National Natural Science Foundation of China Grants (81871302, 81671618, 8150413), the CAMS Initiative for Innovative Medicine (2017-I2M-3-001), and the CAMS Initiative for Innovative Medicine(2017-I2M-B\&R-01).

Compliance with ethical standards The study was approved by the Ethics Committee of the Peking Union Medical College Hospital, and all recruited participants gave informed consent.

\section{Disclosures None.}

Open Access This article is distributed under the terms of the Creative Commons Attribution 4.0 International License (http:// creativecommons.org/licenses/by/4.0/), which permits unrestricted use, distribution, and reproduction in any medium, provided you give appropriate credit to the original author(s) and the source, provide a link to the Creative Commons license, and indicate if changes were made.

\section{References}

1. LeRoy EC et al (1988) Scleroderma (systemic sclerosis): classification, subsets and pathogenesis. J Rheumatol 15(2):202-205 https://www.ncbi.nlm.nih.gov/pubmed/3361530

2. van den Hoogen F, Khanna D, Fransen J, Johnson SR, Baron M, Tyndall A, Matucci-Cerinic M, Naden RP, Medsger TA Jr, Carreira PE, Riemekasten G, Clements PJ, Denton CP, Distler O, Allanore Y, Furst DE, Gabrielli A, Mayes MD, van Laar J, Seibold JR, Czirjak L, Steen VD, Inanc M, Kowal-Bielecka O, Müller-Ladner U, Valentini G, Veale DJ, Vonk MC, Walker UA, Chung L, Collier DH, Csuka ME, Fessler BJ, Guiducci S, Herrick A, Hsu VM, Jimenez S, Kahaleh B, Merkel PA, Sierakowski S, Silver RM, Simms RW, Varga J, Pope JE (2013) 2013 classification criteria for systemic sclerosis: an American College of Rheumatology/ European League against Rheumatism collaborative initiative. Arthritis Rheum 65(11):2737-2747. https://doi.org/10.1002/art. 38098

3. Fernandez-Codina A et al (2017) Cardiac involvement in systemic sclerosis: differences between clinical subsets and influence on survival. Rheumatol Int 37(1):75-84. https://doi.org/10.1007/s00296015-3382-2

4. Morales-Cardenas A et al (2016) Pulmonary involvement in systemic sclerosis. Autoimmun Rev 15(11):1094-1108. https://doi. org/10.1016/j.autrev.2016.07.025

5. Galluccio F et al (2017) Points to consider in renal involvement in systemic sclerosis. Rheumatology (Oxford) 56(suppl_5):v49-v52. https://doi.org/10.1093/rheumatology/kex201

6. Mehra S et al (2013) Autoantibodies in systemic sclerosis. Autoimmun Rev 12(3):340-354. https://doi.org/10.1016/j.autrev. 2012.05.011

7. Okano Y, Steen VD, Medsger TA Jr (1993) Autoantibody reactive with RNA polymerase III in systemic sclerosis. Ann Intern Med 119(10):1005-1013 https://www.ncbi.nlm.nih.gov/pubmed/ 8214977

8. Kuwana M, Kimura K, Kawakami Y (2002) Identification of an immunodominant epitope on RNA polymerase III recognized by systemic sclerosis sera: application to enzyme-linked immunosorbent assay. Arthritis Rheum 46(10):2742-2747. https://doi.org/10. 1002/art.10521

9. Nguyen B, Mayes MD, Arnett FC, del Junco D, Reveille JD, Gonzalez EB, Draeger HT, Perry M, Hendiani A, Anand KK, Assassi S (2011) HLA-DRB1*0407 and *1304 are risk factors for scleroderma renal crisis. Arthritis Rheum 63(2):530-534. https://doi.org/10.1002/art.30111

10. Meyer OC et al (2007) Disease subsets, antinuclear antibody profile, and clinical features in 127 French and 247 US adult patients with systemic sclerosis. J Rheumatol 34(1):104-109 https://www. ncbi.nlm.nih.gov/pubmed/17117486

11. Motegi S, Toki S, Yamada K, Uchiyama A, Ishikawa O (2015) Demographic and clinical features of systemic sclerosis patients with anti-RNA polymerase III antibodies. J Dermatol 42(2):189192. https://doi.org/10.1111/1346-8138.12722

12. Hamaguchi $Y$ et al (2015) Clinical and immunologic predictors of scleroderma renal crisis in Japanese systemic sclerosis patients with anti-RNA polymerase III autoantibodies. Arthritis Rheum 67(4): 1045-1052. https://doi.org/10.1002/art.38994

13. Nikpour M et al (2011) Prevalence, correlates and clinical usefulness of antibodies to RNA polymerase III in systemic sclerosis: a cross-sectional analysis of data from an Australian cohort. Arthritis Res Ther 13(6):R211. https://doi.org/10.1186/ar3544

14. Santiago M, Baron M, Hudson M, Burlingame RW, Fritzler MJ (2007) Antibodies to RNA polymerase III in systemic sclerosis detected by ELISA. J Rheumatol 34(7):1528-1534 https://www. ncbi.nlm.nih.gov/pubmed/17610318 
15. Vitali C, Bombardieri S, Jonsson R, Moutsopoulos HM, Alexander EL, Carsons SE, Daniels TE, Fox PC, Fox RI, Kassan SS, Pillemer SR, Talal N, Weisman MH, European Study Group on Classification Criteria for Sjögren's Syndrome (2002) Classification criteria for Sjogren's syndrome: a revised version of the European criteria proposed by the American-European Consensus Group. Ann Rheum Dis 61(6):554-558

16. Arnett FC et al (1988) The American Rheumatism Association 1987 revised criteria for the classification of rheumatoid arthritis. Arthritis Rheum 31(3):315-324

17. Hochberg MC (1997) Updating the American College of Rheumatology revised criteria for the classification of systemic lupus erythematosus. Arthritis Rheum 40(9):1725. https://doi.org/ 10.1002/1529-0131(199709)40:9\&1t;1725::AID-ART29\&gt;3.0. $\mathrm{CO} ; 2-\mathrm{Y}$

18. Bohan A, Peter JB (1975) Polymyositis and dermatomyositis (first of two parts). N Engl J Med 292(7):344-347. https://doi.org/10. 1056/nejm197502132920706

19. Bohan A, Peter JB (1975) Polymyositis and dermatomyositis (second of two parts). N Engl J Med 292(8):403-407. https://doi.org/ 10.1056/nejm197502202920807

20. Sobanski V et al (2014) Prevalence of anti-RNA polymerase III antibodies in systemic sclerosis: New data from a French cohort and a systematic review and meta-analysis. Arthritis Rheum 66(2):407-417. https://doi.org/10.1002/art.38219

21. Koenig M, Dieude M, Senecal JL (2008) Predictive value of antinuclear autoantibodies: the lessons of the systemic sclerosis autoantibodies. Autoimmun Rev 7(8):588-593. https://doi.org/10.1016/ j.autrev.2008.06.010

22. Steen VD (2005) Autoantibodies in systemic sclerosis. Semin Arthritis Rheum 35(1):35-42. https://doi.org/10.1016/j.semarthrit. 2005.03.005

23. Elhai M, Meune C, Avouac J, Kahan A, Allanore Y (2012) Trends in mortality in patients with systemic sclerosis over 40 years: a systematic review and meta-analysis of cohort studies. Rheumatology (Oxford) 51(6):1017-1026. https://doi.org/10. 1093/rheumatology/ker269

24. Ooi C et al (2018) Mortality in the Waikato Hospital Systemic Sclerosis Cohort. Int J Rheum Dis 21(1):253-260. https://doi.org/ 10.1111/1756-185x.13111

25. Woodworth TG, Suliman YA, Li W, Furst DE, Clements P (2016) Scleroderma renal crisis and renal involvement in systemic sclerosis. Nat Rev Nephrol 12(11):678-691. https://doi.org/10.1038/ nrneph.2016.124
26. Kuwana M (2017) A to-do list at diagnosis of systemic sclerosis with positive anti-rna polymerase III antibodies. J Rheumatol 44(5): 550-552. https://doi.org/10.3899/jrheum.170037

27. Hudson M, Baron M, Tatibouet S, Furst DE, Khanna D, International Scleroderma Renal Crisis Study Investigators (2014) Exposure to ACE inhibitors prior to the onset of scleroderma renal crisis-results from the International Scleroderma Renal Crisis Survey. Semin Arthritis Rheum 43(5):666-672. https://doi.org/10. 1016/j.semarthrit.2013.09.008

28. Steen VD, Medsger TA Jr (1998) Case-control study of corticosteroids and other drugs that either precipitate or protect from the development of scleroderma renal crisis. Arthritis Rheum 41(9): 1613-1619. https://doi.org/10.1002/1529-0131(199809)41: 9<1613::AID-ART11>3.0.CO;2-O

29. Iudici $\mathrm{M}$ et al (2013) Glucocorticoids in systemic sclerosis: weighing the benefits and risks - a systematic review. Clin Exp Rheumatol 31(2 Suppl 76):157-165 https://www.ncbi.nlm.nih. gov/pubmed/23910618

30. Kuwana M, Okano Y, Pandey JP, Silver RM, Fertig N, Medsger TA Jr (2005) Enzyme-linked immunosorbent assay for detection of anti-RNA polymerase III antibody: analytical accuracy and clinical associations in systemic sclerosis. Arthritis Rheum 52(8):24252432. https://doi.org/10.1002/art.21232

31. Ingraham KM, O'Brien MS, Shenin M, Derk CT, Steen VD (2010) Gastric antral vascular ectasia in systemic sclerosis: demographics and disease predictors. J Rheumatol 37(3):603-607. https://doi.org/ 10.3899/jrheum.090600

32. Ceribelli A, Cavazzana I, Airò P, Franceschini F (2010) Anti-RNA polymerase III antibodies as a risk marker for early gastric antral vascular ectasia (GAVE) in systemic sclerosis. J Rheumatol 37(7): 1544. https://doi.org/10.3899/jrheum.100124

33. Hung EW, Mayes MD, Sharif R, Assassi S, Machicao VI, Hosing C, St Clair EW, Furst DE, Khanna D, Forman S, Mineishi S, Phillips K, Seibold JR, Bredeson C, Csuka ME, Nash RA, Wener MH, Simms R, Ballen K, Leclercq S, Storek J, Goldmuntz E, Welch B, Keyes-Elstein L, Castina S, Crofford LJ, Mcsweeney P, Sullivan KM (2013) Gastric antral vascular ectasia and its clinical correlates in patients with early diffuse systemic sclerosis in the SCOT trial. J Rheumatol 40(4):455-460. https://doi.org/10.3899/ jrheum. 121087

Publisher's note Springer Nature remains neutral with regard to jurisdictional claims in published maps and institutional affiliations. 